\title{
Tryptophan Depletion Disrupts the Motivational Guidance of Goal-Directed Behavior as a Function of Trait Impulsivity
}

\author{
Roshan Cools*, I,4, Andrew Blackwell', Luke Clark', Lara Menzies', Sylvia Cox ${ }^{3,5}$ and Trevor W Robbins' \\ 'Department of Experimental Psychology, University of Cambridge, Cambridge, UK; ${ }^{2}$ Department of Psychiatry, University of Cambridge, \\ Addenbrooke's Hospital, Cambridge, UK; ${ }^{3}$ MRC Cognition and Brain Sciences Unit, Cambridge, UK; ${ }^{4}$ Department of Psychology, Helen Wills \\ Neuroscience Institute, University of California, Berkeley, CA, USA; ${ }^{5}$ Institute of Brain and Behavior, University of Maastricht, Maastricht, \\ The Netherlands
}

\begin{abstract}
Serotonin (5-HT) is well known to affect the motivational properties of stimuli predictive of rewards as well as the inhibitory control of behavior. Here, central 5-HT depletion was induced by the acute tryptophan (TRP) depletion (ATD) procedure in young healthy volunteers to examine the role of 5-HT in motivated action and prepotent response inhibition. A novel reaction-time task, tailored to individual differences in general cognitive speed, was employed to measure the guidance of behavior by motivationally relevant signals predictive of reinforcement likelihood, while the stop-signal reaction-time task was used to measure response inhibition. Following the TRP-balancing control drink, cues predictive of high-reinforcement certainty induced faster, but less accurate responses compared with cues predictive of lower reinforcement certainty. Depletion of central 5-HT modulated this coupling between motivation and action by slowing responses and increasing accuracy as a function of incentive certainty. These effects of ATD on motivated action correlated highly with individual differences in the personality trait of Nonplanning Impulsiveness (Barratt Impulsivity Scale (BIS-I I)), so that strongest effects on motivated action were observed in high-impulsive individuals. By contrast, ATD left unaltered the ability to inhibit prepotent responses. Our findings may have implications for a variety of neuropsychiatric disorders including impulsive aggressive disorders and depression.
\end{abstract}

Neuropsychopharmacology (2005) 30, 1362-1373, advance online publication, I6 March 2005; doi: I 0. I038/sj.npp. I 300704

Keywords: impulsivity; serotonin; reward certainty; motivation; self-control; tryptophan depletion

\section{INTRODUCTION}

Incentive motivation, or the attraction to external stimuli that have appetitive or rewarding properties, is central to many normal and abnormal behaviors (Robbins and Everitt, 2003; Robinson and Berridge, 2003). It enables the attainment of goals and appropriate behavioral (and autonomic and endocrine) adaptation to environmental change, and may also contribute to the symptomatology of abnormal states such as depression, impulsive-aggressive behavior, attention-deficit/hyperactivity disorder, and drug addiction.

Serotonin (5-HT) has been extensively implicated in depressed mood and is well known to affect the motivational properties of stimuli predictive of rewards (Fletcher et al, 1999; Sasaki-Adams and Kelley, 2001; Deakin, 1983; Wilkinson et al, 1995; Graeff et al, 1986), probably through

\footnotetext{
*Correspondence: Dr R Cools, Helen Wills Neuroscience Institute, I 32 Barker Hall, Berkeley, CA 94720-3190, USA, Tel: + I 510642 2839, Fax: + I 510642 3192, E-mail: roshanco@berkeley.edu Received II May 2004; revised 27 October 2004; accepted 29 December 2004

Online publication: 24 January 2005 at http://www.acnp.org/citations/ NPPO I 2405040225/default.pdf
}

interaction with the mesolimbic dopamine (DA) system (Robbins et al, 1989; Robbins and Everitt, 2002). Treatment with selective serotonin reuptake inhibitors (SSRIs) dose dependently decreases brain self-stimulation thresholds (Harrison and Markou, 2001), while the reinforcing effects of cocaine, cocaine-associated stimuli, and other stimuli predictive of rewards are potentiated by SSRI treatment and, conversely, attenuated by central 5-HT depletion (Aronson et al, 1995; Sasaki-Adams and Kelley, 2001; Redgrave and Horrell, 1976).

A cardinal feature of depression is reduced motivation (anhedonia, apathy), where neuropsychological studies have further highlighted the relevance of reinforcement processing and an association with failures to activate appetitive behavior upon presentation of reward cues (Hughes et al, 1985; Henriques et al, 1994). Acute reduction of central 5-HT function through dietary depletion of tryptophan (TRP), a precursor of 5-HT (acute TRP depletion, ATD), can reinstate depressive symptoms and flattened affect (Young et al, 1985). In addition, SSRIs exert antidepressant effects.

In keeping with the hypothesis that $5-\mathrm{HT}$ is critically involved in the processing of incentive signals, studies in humans have shown that depletion of central 5-HT impairs 
decision-making in gambling tasks, visual discrimination, and reversal learning, possibly due to deficits in (re)acquiring associations between stimulus and reward values (Park et al, 1994; Rogers et al, 1999, 2003; Murphy et al, 2002). A recent study revealed that 5-HT depletion in healthy volunteers impaired the ability to discriminate between large and small rewards (Rogers et al, 2003). Based on these observations, the authors proposed that 5-HT depletion alters decision-making by impairing the processing of reward cues.

5-HT neurotransmission has been implicated not only in the processing of incentive-motivational signals but also in the inhibitory control of behavior (Soubrie, 1986; Evenden, 1999). Like depression, impulsive pathology has been associated with a deficiency of central 5-HT. CSF concentrations of the 5-HT metabolite 5-HIAA correlate inversely with impulsive behaviors in many neuropsychiatric and personality disorders including aggressive, conduct, and attention-deficit/hyperactivity disorders (Evenden, 1999). Furthermore, recent studies with human volunteers have found that ATD increases impulsive and aggressive behavior, although these effects appear to depend on individual differences in impulsive personality traits (Cherek and Lane, 1999; LeMarquand et al, 1999; Dougherty et al, 1999; Bjork et al, 2000; Marsh et al, 2002; Crean et al, 2002). Findings from studies with clinical human populations are corroborated by an extensive number of animal studies, which suggest that impulsive choice in delaydiscounting paradigms and premature responding in choice reaction-time tasks implicate changes in the 5 -HT system (Wogar et al, 1993; Harrison et al, 1997a, b; Puumala and Sirvio, 1998; Koskinen et al, 2000; Mobini et al, 2000; Dalley et al, 2002; Passetti et al, 2003; Winstanley et al, 2003; Liu et al, 2004).

In this study, we used ATD, a well-recognized method for directly reducing central nervous system 5-HT (Nishizawa et al, 1997; Carpenter et al, 1998), in healthy human volunteers to examine effects of central 5-HT depletion in healthy volunteers in the domains of incentive motivation and the inhibitory control of behavior. First, a novel reaction-time paradigm, tailored to individual differences in cognitive speed, was employed in which reward probability was varied systematically. A cue stimulus determined the (noncontingent) probability of reinforcement, while the direction and magnitude of reinforcement was contingent upon the accuracy and speed of a response in an odd-oneout choice task. Thus, the task measured the ability to adapt responding according to the incentive-motivational value of the cue stimulus. Second, we administered a well-validated measure of response inhibition (the stop-signal reactiontime task (SSRT); Logan et al, 1984; Aron et al, 2003) and a self-report measure of trait impulsivity, the Barratt Impulsivity Scale (BIS-11; Patton et al, 1995) to compare effects on motivated action with effects on response inhibition.

On the basis of the above-reviewed evidence for diminished 5-HT function in depression, we predicted that reduced central 5-HT would induce a loss of motivated action in the novel reaction-time task. In addition, we hypothesized that decreased 5-HT availability would impair the ability to inhibit prepotent responding on the SSRT. Moreover, we predicted that the effect on response inhibition would be a function of trait impulsivity as measured with the BIS-11, with greatest ATD-induced effects in high-impulsive subjects.

\section{METHODS}

\section{Subjects}

Procedures were approved by the Local Research Ethical Committee in Cambridge and were in accord with the Helsinki Declaration of 1975. Twelve young, healthy male (right-handed) volunteers were recruited for participation in a combined functional magnetic resonance imaging (subjects performed a reversal learning experiment in the scanner; data not reported here; Evers et al, 2005) and neuropsychological crossover, placebo-controlled experiment. An additional sample of 12 young, healthy male volunteers was recruited for participation in a complementary single-session neuropsychological experiment (six $\mathrm{TRP}+$ and six TRP-). This additional sample was necessary to obtain sufficient statistical power for between-subject comparisons of first-session data, not contaminated by practice effects. The latter sample also served as a reserve sample for the imaging experiment. All subjects were screened for psychiatric and neurological disorders, gave written informed consent, and were paid for participation. Exclusion criteria were any history of cardiac, hepatic, renal, pulmonary, neurological, psychiatric or gastrointestinal disorders, medication use, and history of major depression or bipolar affective disorder.

Neuropsychological data on the primary task of interest (the cued-reinforcement reaction-time task (CRRT)) were acquired for 10 out of 12 scanned subjects and 11 out of 12 reserve subjects. Two subjects did not complete the task (one scanned, one reserve) and another scanned subject was classified as an outlier in terms of his mean response time (RT) on the CRRT: ( $>3$ standard deviation (SD) larger than the group mean RT). This resulted in a sample size of 10 for the within-subject comparison (mean age: $23.8 \pm 2.8$ ) and a size of 22 for the between-subject 'first-session' comparison (TRP $+: n=12$; mean age: 23.8 , SD: 2.6 ; and TRP $-: n=10$; mean age: 23.3, SD: 3.9). Sample size for the within-subject analysis of the SSRT was 11 (the subject who was classified as an outlier on CRRT was also an outlier on the SSRT) and sample size for the between-subject analysis was 23 .

Procedures are described for the scanned subjects only. Procedures for reserve subjects were identical, but they were assessed only once, following either TRP + or TRP-, and were not scanned.

\section{General Procedure}

Using the ATD procedure, central TRP was depleted by ingesting an amino-acid load that does not contain TRP but does include other large neutral amino acids (LNAAs) (Reilly et al, 1997). The reduction is achieved by increasing protein synthesis in the liver with subsequent decrease of plasma TRP stores. In addition, the amino-acid load results in competition for the active transport system that the amino acids share for entry across the blood-brain barrier, resulting in reduced availability of TRP in the brain. 
Participants were scanned and subsequently assessed on a neuropsychological battery at the Wellcome Trust Clinical Research Facility (CRF) in Addenbrooke's Hospital on two separate test sessions, separated by at least 1 week. Sessions were approximately counterbalanced according to a doubleblind crossover design with an experimental and a placebo condition. However, due to subject dropout on the CRRT (see above), three out of 10 subjects received TRP - on their first session, while seven out of 10 subjects received TRP + on their first session. Test order did not confound the results as evidenced by the between-subject comparison of first-session data. Volunteers were asked to abstain from alcohol, caffeine, and high-carbohydrate food for $24 \mathrm{~h}$ prior to the test session. During the test days, they followed a low protein diet. In the morning or early afternoon of a test day (0800, 1000, 1200 or 1400), volunteers arrived at the CRF, where (i) Visual Analogue Scales (VAS) (measuring mood, nausea and anxiety) were administered, (ii) a blood sample was taken, and (iii) nutritionally balanced (TRP + ) or a TRP-free (TRP-) amino-acid drink was ingested. During the ingestion of the drink on the first visit, subjects completed self-report questionnaires. Following a resting period of approximately $5 \mathrm{~h}$ (mean, SD for TRP-: $4.5 \mathrm{~h}$, $35 \mathrm{~min}$; TRP + : $5 \mathrm{~h}, 40 \mathrm{~min}$ ) to ensure stable and low TRPlevels, participants entered the scanner. After completion of the scanning sessions, subjects were guided to the CRF, where following a second blood sample, they performed a 1.5-h-long neuropsychological test battery (starting approximately $6.5 \mathrm{~h}$ after ingestion of the mixture). The SSRT (see Aron et al, 2003) and the novel CRRT task were administered in the same order on each occasion in each subject.

One subject fainted during the baseline blood test but recovered quickly. Another subject vomited approximately $2 \mathrm{~h}$ following ingestion of the TRP + drink. Other subjects reported no side effects apart from transient nausea.

\section{Amino-Acid Mixtures}

The TRP - drink contained a total of $75 \mathrm{~g}$ of different amino acids, with the amounts of each specific amino acid according to the proportions described by Young et al (1985): $4.1 \mathrm{~g}$ L-alanine, $2.4 \mathrm{~g}$ glycine, $2.4 \mathrm{~g}$ L-histidine, $6.0 \mathrm{~g} \mathrm{~L}$ isoleucine, $10.1 \mathrm{~g}$ L-leucine, $6.7 \mathrm{~g}$ L-lysine, $4.3 \mathrm{~g}$ L-phenylalanine, $9.2 \mathrm{~g}$ L-proline, $5.2 \mathrm{~g}$ L-serine, $4.3 \mathrm{~g}$ L-threonine, $5.2 \mathrm{~g}$ L-tyrosine, $6.7 \mathrm{~g}$ L-valine, $3.7 \mathrm{~g}$ L-arginine, $2.0 \mathrm{~g}$ L-cysteine, and $3.0 \mathrm{~g} \mathrm{~L}$-methionine. The TRP + drink contained the same amino acids, plus $3.0 \mathrm{~g}$ TRP. The drinks were prepared by adding $200 \mathrm{ml}$ tap water with the addition of lemon-lime or grapefruit flavoring to compensate for the unpleasant taste.

\section{Self-Report Measurements}

VAS containing the items drowsy, sad, happy, anxious, and nauseous were administered five times during the test day. The Barratt Impulsiveness Scale (BIS-11; Patton et al, 1995) was completed $1 \mathrm{~h}$ after ingestion of the drink. Total BIS-11 scores ranged from 50 to 72 (mean: 62.7; SD: 7.2). No effects of ATD on mood and health were found (data not shown here, see Evers et al, 2005).

\section{Cued-Reinforcement Reaction-Time Task}

The task was programmed in Microsoft Visual Basic 6.0 and presented on a Toshiba Satellite laptop (14" monitor).

On each trial, subjects were presented with three horizontally adjacent circles (see Figure 1). Within each of these three circles, a smaller circle was presented. Subjects were asked to identify the 'odd-one-out' circle. Stimuli were based on the 'circles task' used by Duncan et al (2000). Responses were made by pressing, with the right index, middle or ring finger, one of three keys on an English keyboard (' $<$ ', ' $>$ ' or '?') corresponding to the location of the 'odd-one-out' circle. An odd-one-out task rather than a simple RT task was employed because the task was expected to induce greater variability in subjects' RTs and to create response uncertainty (Kagan, 1966).

On some trials (see below), feedback coincided with the response. Following a correct response, a green smiley face and an increase in total point score (see Figure 1) was presented in the center of the screen (for $500 \mathrm{~ms}$ ). Following an incorrect response, a red sad face was presented. The number of points gained was dependent on accuracy and $\mathrm{RT}$, so that a fast correct response was rewarded with 100 points (and a flourish sound), a slow correct response with 1 point (and a short high-frequency tone), and an incorrect response with 0 points (and a low-frequency tone). Thus, while subjects were rewarded with a green smiley face, a high-frequency tone (or flourish) and points for a correct response, they were punished with a red sad face and a low-frequency tone for an incorrect response. Incorrect responses did not result in loss of points. The cutoff for a 'fast response' was calculated based on a practice block that directly preceded the experimental blocks (see below). The total point score remained on the screen throughout the task. On 'nonfeedback' trials, responses were not followed by any feedback, subjects could not win any points, and the stimulus was replaced by a fixation cross.

The feedback face (or fixation cross) was replaced by a blue, red, or yellow rectangular stimulus window $\left(13 \times 26 \mathrm{~cm}^{2}\right)$, which cued the probability of receiving reinforcement on the next trial. The three different color cues were associated with 10,50, and 90\% reinforcement probability. The next circles-stimulus was presented a variable interval after the presentation of the colored stimulus window, so that the interstimulus interval remained constant at $2500 \mathrm{~ms}$. The stimulus remained on the screen until a response was made or until a 'too late' message was displayed (if a response was not made within $2000 \mathrm{~ms}$ of stimulus onset).

Subjects were told that they had to obtain as many points as possible.

Prior to the experimental blocks, two practice blocks of 20 trials were given, in which circles-stimuli were presented without colored stimulus windows (and without feedback). Subjects were encouraged to respond as fast as possible without making any mistakes. A mean RT and SD were extracted from the second practice block (provided the percentage of correct response exceeded 85\%) and a cutoff score was calculated by subtracting the SD from the mean RT. For example, with a mean of $600 \mathrm{~ms}$ and a SD of $120 \mathrm{~ms}$, RTs faster than $480 \mathrm{~ms}$ would be rewarded with 100 points. 
Cue

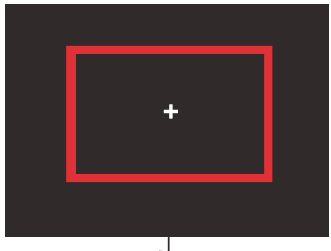

Stimulus

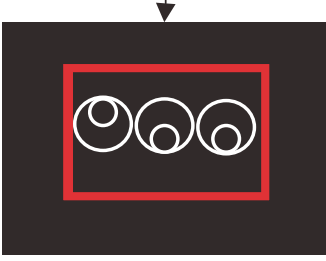

Response
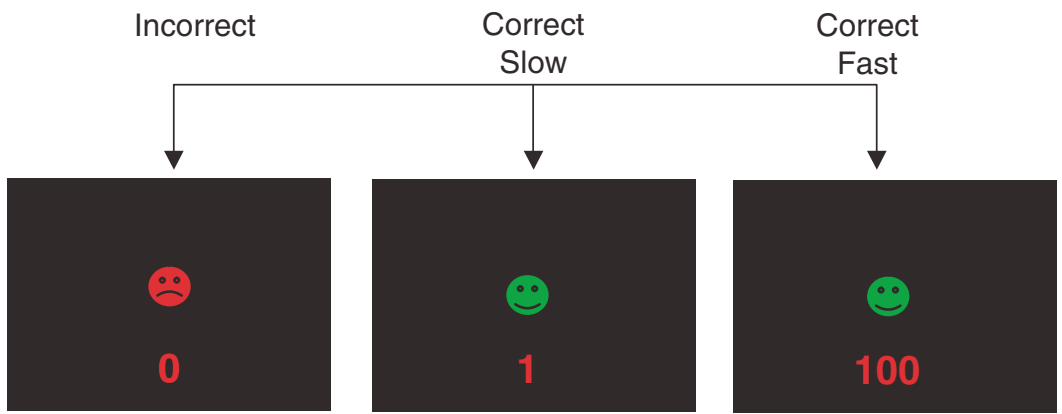

Figure I Trial structure of a reinforced trial from the cued CRRT. Subjects were required to make one of three responses corresponding to the 'oddone-out' circle, as fast as possible without making mistakes. A colored stimulus window indicated the probability of receiving reinforcement (I 00 points and a smiley face for a response faster than the cutoff score, I point and a smiley face for a response slower than the cutoff score, and 0 points and a sad face for an incorrect response). No feedback was presented and no points were obtained on the remaining proportion of trials. Reinforcement probabilities varied parametrically between 10,50, and 90\% (depending on the color of the stimulus window) and subjects were encouraged to gain as many points as possible.

Two blocks of 96 trials were administered. In each block, there were 32 trials of each cue type and 12 different circlesstimulus exemplars, where each exemplar consisted of three outer circles (diameter: $5 \mathrm{~cm}$ ) and three inner circles (diameter: $2.5 \mathrm{~cm}$ ). Stimulus exemplars, responses, and response repetitions were counterbalanced across cue type, so that no stimulus repetitions and no more than two response repetitions could occur. Colors of stimulus window were always red, blue, and yellow. The mapping between the three colors and the three reinforcement probabilities was counterbalanced across subjects and mixture, so that five subjects received mapping 1 after ingestion of the depleting mixture and mapping 2 after the balancing mixture, while this mapping order was reversed for the other five subjects.

At post-test, subjects were given a de-brief questionnaire on which they indicated their estimate of reinforcement probability for each color, using $10 \mathrm{~cm}$ analogue scales. This provided a measure of explicit awareness of stimulusreinforcement contingencies.

\section{Stop-Signal Reaction-Time Task (Logan et al, 1984; Aron et al, 2003)}

On this classic paradigm of prepotent response inhibition, subjects were required to make a speeded response on 'go' trials (left response for left-pointing arrow, right response for right-pointing arrow), but to withhold their response on 'stop' trials (signaled by a $300 \mathrm{~Hz}$ tone). Stopping was made difficult by having a preponderance of 'go' trials (75\%). The timing of the stop-signal was manipulated by means of a tracking algorithm (Osman et al, 1990) in such a way as to allow estimation of SSRT. Subjects performed five blocks of 64 trials each, and were given visual feedback after each block for their average correct 'go' reaction time and the number of discrimination errors made (incorrect responses on 'go' trials). Subjects were not given feedback with regard to successful or failed inhibition, but were urged to do their best to stop, while continuing to respond as fast as possible on 'go' trials.

\section{Data Analysis}

All data were analyzed using SPSS v11.0. (SPSS Inc., Chicago, IL).

Biochemical measures. Blood (venous) samples (10 ml) were taken immediately before ingestion of the amino-acid drink and after the scanning session, approximately six and a half hours after administration, to determine the plasma TRP level, and the TRP/ $L$ LNAA ratio. This ratio was calculated from the serum concentrations of total TRP divided by the sum of the LNAAs (tyrosine, phenylalanine, valine, isoleucine, leucine) and is important because the uptake of TRP in the brain is strongly associated with the amounts of other competing LNAAs (TRP and the other LNAA share the blood-brain barrier). Venous samples were taken in lithium heparin tubes and stored at $-20^{\circ} \mathrm{C}$. Plasma TRP concentrations were determined by an isocratic high-performance liquid chromatography (HPLC) method 
of analysis. Plasma proteins were removed by precipitation with 3\% trichloroacetic acid and centrifugation at 3000 revs, $4{ }^{\circ} \mathrm{C}$ for $10 \mathrm{~min}$, and then pipetted into heparin aliquots. An aliquot was diluted in mobile phase before injection onto the HPLC analytical column. Fluorescence end-point detection was used to identify TRP.

Cued-reinforcement reaction-time task. Incorrect trials and trials on which subjects did not respond within the maximum of $2000 \mathrm{~ms}$ (omissions) were excluded from RT analyses. Mean proportions of errors and omissions were arcsin transformed ( $2 \arcsin \sqrt{ } \times$; Howell, 1997, p 328). Two separate analyses were performed: (i) a within-subject analysis $(n=10)$ and a between-subject analysis on data acquired on a first session only $(n=22)$. Data were analyzed using repeated measures ANOVAs with either three withinsubject factors (drink: TRP + vs TRP-; block: 1 vs 2; and cue: 10 vs 50 vs 90\%) or one between-subject factor (drink) and two within-subject factors (block and cue) and a priori defined contrasts (cue $10 \%$ vs cue $90 \%$; cue $10 \%$ vs cue $50 \%$; cue $50 \%$ vs cue $90 \%$ ). Greenhouse-Geisser corrections were applied when the sphericity assumption was violated. In crossover designs, the index of variation commonly used for within-subject comparisons is not the standard error of the mean (SEM), but the standard error of the difference between means (SED). The SED is calculated using the formula $\mathrm{SED}=\sqrt{ }[(2 \times \mathrm{MSe}) / N]$, where $\mathrm{MSe}$ is the mean square for the residual term and $N$ is the number of observations (Cochran and Cox, 1957). The SED is thought to be less appropriate as a variability index for betweensubject comparisons, so the SEM was computed instead. The statistical threshold was $p<0.05$.

Stop-signal reaction-time task. Four measures were computed: the SSRT, the probability of inhibiting a response over the whole of the task, mean RT on GO trials, and the number of discrimination errors on GO trials. The reader is referred to Aron et al (2003) for further details. Two separate analyses were performed: (i) a within-subject analysis $(n=11)$ and a between-subject analysis on data acquired on a first session only $(n=23)$. Data were analyzed using paired $t$-tests or independent sample $t$-tests. The statistical threshold was $p<0.05$.

Correlations. Difference scores were calculated (from the crossover, within-subject data only) reflecting ATD-induced performance changes on the CRRT and SSRT tasks. Reinforcement-induced 'speeding' scores were obtained by subtracting (i) RTs on the 90\%-reinforced trials from RTs on the $50 \%$-reinforced trials, (ii) RTs on the $50 \%$-reinforced trials from RTs on the 10\%-reinforced trials, and (iii) RTs on the $90 \%$-reinforced trials from RTs on the $10 \%$ reinforced trials. Similarly, reinforcement-induced 'accuracy-increase' scores were obtained by subtracting (i) the proportion of correct responses on the 50\%-reinforced trials from that on the $90 \%$-reinforced trials, (ii) the proportion of correct responses on 10\%-reinforced trials from that on the 50\%-reinforced trials, and (iii) the proportion of correct responses on 10\%-reinforced trials from that on the $90 \%$-reinforced trials. The ATD-induced performance change scores were calculated by subtracting these reinforcement-induced 'speeding' and 'accuracyincrease' measures following TRP + from the same values following TRP - . The same was done for the stop-signal task measure of SSRT. Spearman's nonparametric correlation coefficients were calculated between these ATD-induced changes in behavior on the CRRT and SSRT tasks and scores on the three second-order factors of the BIS-11 (Patton et al, 1995). Spearman (Rs) rather than Pearson correlation coefficients were computed because not all scores from the BIS-11 were normally distributed in the population sampled as evidenced by a KolmogorovSmirnov test of normality. The lack of orthogonality between variables (eg between RT and accuracy) renders a standard Bonferroni correction for multiple comparisons invalid. Therefore, all relevant correlations reported to be significant here $(p<0.05)$ are plotted to allow inspection.

\section{RESULTS}

\section{Biochemical Measures}

Repeated measures ANOVA of the 'within-subject' data revealed a significant two-way interaction of treatment drink by time of blood test, due to significant reductions in total TRP - levels $\left(\mathrm{F}_{1,9}=47.6, p<0.0001\right)$ and TRP/ $\Sigma$ LNAA ratios $\left(\mathrm{F}_{1,9}=8.0, p=0.02\right) 6.5 \mathrm{~h}$ following $\mathrm{TRP}-$ relative to $\mathrm{TRP}+($ see Figure 2a). Analyses of simple effects (ie the effects of certain variables at the specific level of another variable; Howell, 1997, p 412) revealed significant effects of drink at $6.5 \mathrm{~h}$ in terms of both total TRP $\left(t_{9}=-8.0\right.$, $p<0.0001)$ and ratios $\left(t_{9}=-4.0, p=0.003\right)$, but no effects at baseline (total TRP: $t_{9}=0.4, p=0.7$; ratio: $t_{9}=-1.2$, $p=0.25)$. Similarly, analysis of the first-session data also revealed a significant two-way interaction between drink group and time of test for both the total TRP- levels $\left(\mathrm{F}_{1,20}=56.3, p<0.0001\right)$ and the ratios $\left(\mathrm{F}_{1,20}=20.5\right.$, $p<0.0001$ ) (see Figure 2b).

\section{Cued-Reinforcement Reaction-Time Task}

Data from the within-subject analysis are presented in Figure $3 \mathrm{a}$ and $\mathrm{b}$ and data from the between-subject analysis in Figure $3 c$ and $d$. Inspection of the data by eye reveals that, following the control drink, subjects responded faster and made more errors on high-reinforcement trials than on low- (and medium-) reinforcement trials. ATD abolished this reinforcement-induced speeding and reversed the error pattern, so that, following TRP-, subjects did not exhibit reinforcement-induced speeding and made fewer errors on high-reinforcement than low-reinforcement trials.

Between-subject ANOVAs of RT data from the subjects' first visits revealed a highly significant omnibus group by cue interaction $\left(\mathrm{F}_{2,40}=5.9, p=0.009\right)$ as well as two significant planned contrasts $\left(90-10 \%: \mathrm{F}_{1,20}=7.9, p=0.011\right.$ and $\left.90-50 \%: \mathrm{F}_{1,20}=13.9, p=0.001\right)$. Simple effects analyses revealed that these effects were due to subjects responding faster on $90 \%$-reinforced trials than on both the $10 \%$ - and 50\%-reinforced trials following TRP + (90-10\%: $\left.\mathrm{F}_{1,11}=18.4, p=0.001 ; 90-50 \%: \mathrm{F}_{1,11}=15.8, p=0.002\right)$, but not following TRP-(90-10\%: $\mathrm{F}_{1,9}=1.8, p=0.2 ; 90-50 \%$ : $\left.\mathrm{F}_{1,9}=0.9, p=0.4\right)$. There was no main effect of drink group $\left(F_{1,20}=0.5, p=0.5\right)$. Analysis of the error data revealed only 
nonsignificant tendencies and some significant simple effects, and these are presented in the legends of Figure $3 \mathrm{c}$ and $\mathrm{d}$.

Repeated measures ANOVAs with ATD as a withinsubject factor revealed only nonsignificant tendencies toward interactions between ATD and reinforcement cue. Subsequent analyses revealed several significant simple effects, which are described in the legends of Figure $3 a$ and $b$.
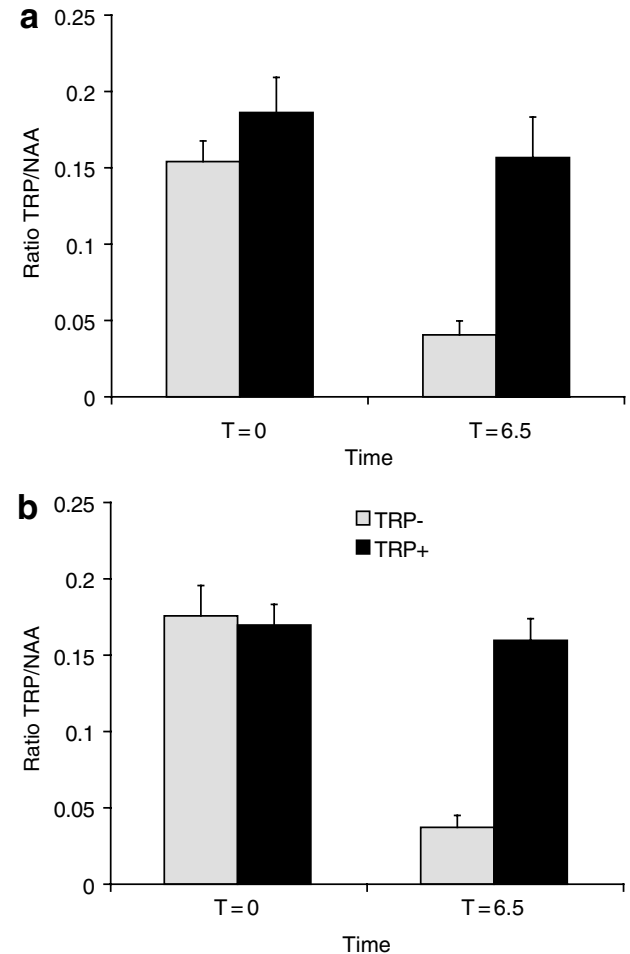

Figure $2 \mathrm{TRP} / \Sigma \mathrm{LNAA}$ ratios at baseline $(t=0)$ and $6.5 \mathrm{~h}$ after intake of the mixture: (a) within-subject analysis and (b) between-subject analysis. Error bars represent SEM.

Figure 3 (a) Mean RT values from the within-subject comparison as a function of anticipated reinforcement certainty and amino-acid drink (TRP levels). Simple effect analyses indicated that subjects responded faster on 90\%-reinforced trials than on 50\%-reinforced trials following TRP + $\left(F_{1,9}=8.4, p=0.018\right)$, but not following TRP- $\left(F_{1,9}=0, p=0.9\right)$. Error bars represent SED. (b) Mean proportion of correct responses from the within-subject comparison. Simple effect analyses revealed that subjects made more correct responses on $90 \%$-reinforcement trials than on $10 \%$ reinforcement following TRP $-\left(F_{1,9}=8.7, p=0.016\right)$, but not following TRP $+\left(F_{1,9}=0.09, p=0.8\right)$. Errors bars represent SED. (c) Mean RT values from the between-subject comparison. Statistically significant effects are described in the text. Error bars represent SEM. (d) Mean proportion of correct responses from the between-subject comparison. Simple interaction effects analysis revealed a significant group by cue interaction in block I $\left(F_{2,40}=4 . I, p=0.031\right)$, but not in block $2\left(F_{2,40}=0.15, p=0.86\right)$. In block I, TRP + subjects made more errors on $90 \%$-reinforcement trials than on $10 \%$-reinforcement trials $\left(F_{1,1}=6.4, p=0.028\right)$, while, in contrast, TRPsubjects made less errors on $90 \%$-reinforcement trials than on $10 \%$ reinforcement trials $\left(F_{1,9}=6.2, p=0.036\right)$. Data shown are from both sessions. Error bars represent SEM.
Correlations. Correlational analyses revealed a strong dependency of CRRT performance and its modulation by ATD on individual differences in the personality trait of nonplanning impulsivity (as measured with BIS-11). These correlations revealed that effects of ATD were profound only in subjects who reported high impulsivity and suggest
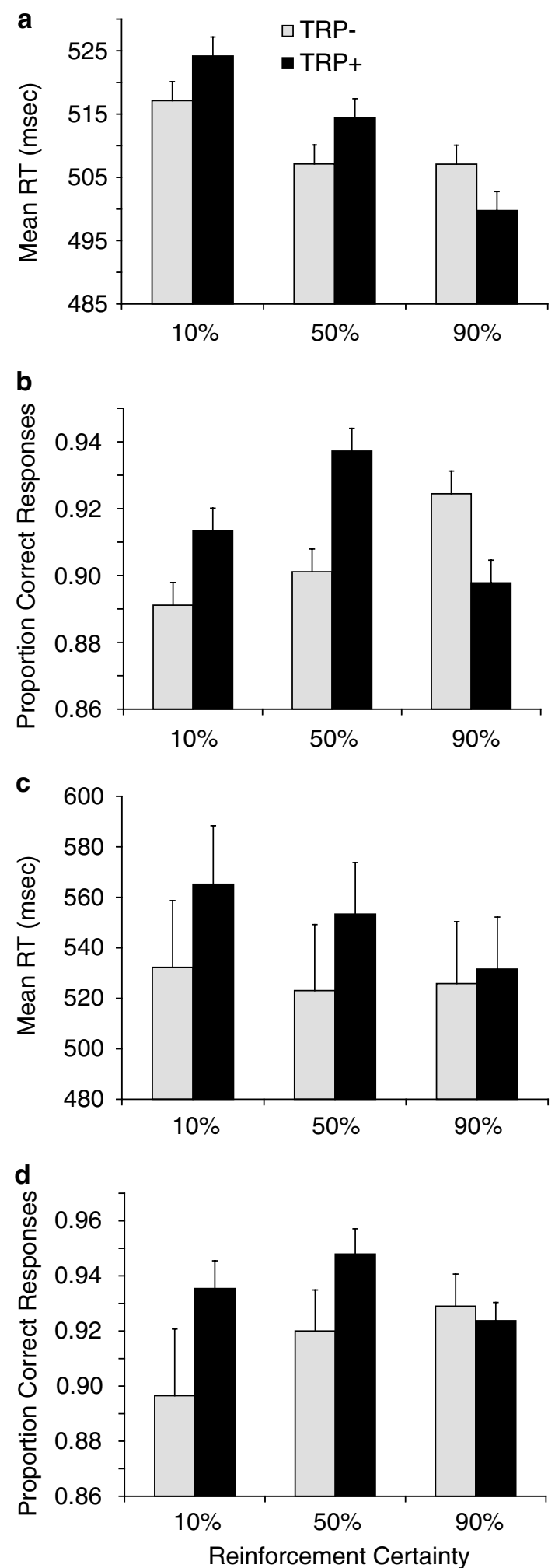

Neuropsychopharmacology 
that heterogeneity in terms of individual differences in trait impulsivity account for the marginal effects of ATD over the group as a whole.

Thus, a significant negative correlation was observed between the second-order factor Nonplanning Impulsiveness of the BIS-11 and the ATD-induced reduction in reinforcement-induced speeding on $90 \%$-reinforced trials compared with 50\%-reinforced trials $\left(\mathrm{Rs}_{10}=-0.81\right.$, $p=0.004)$, primarily due to a strong negative relationship with the primary BIS factor III, lack of self-control $\left(\mathrm{Rs}_{10}=-0.9, p<0.0001\right)$ (Figure 4$)$. This highly significant correlation reflects the finding that ATD reduced reinforcement-induced speeding to a greater extent in more impulsive subjects. Inspection of the data and post hoc correlational analysis reveals that there was a significant positive relationship between lack of self-control and reinforcement-induced speeding following the balancing $\mathrm{TRP}+$ drink $\left(\mathrm{Rs}_{10}=0.8, p=0.005\right)$, but a nonsignificant negative relationship between lack of self-control and reinforcement-induced speeding following the depleting TRP - drink $\left(\mathrm{Rs}_{10}=-0.56, p=0.1\right)$ (inset Figure 4). A Williams test of the difference between these two nonorthogonal correlations revealed that the two correlations were significantly different $\left(t_{7}=4.44, p<0.01\right)$ (Howell, 1997, p 263). Cues predictive of high-reinforcement certainty induced greater speeding in high-impulsive subjects relative to low-impulsive subjects following the control drink. In contrast, following the TRP-depleting drink, the same high-reinforcement cues actually induced, if anything, greater slowing in high-impulsive subjects relative to low-impulsive subjects. Furthermore, the improvement in reinforcement-induced accuracy on $90 \%$ trials compared with $50 \%$ trials, induced by ATD, also correlated significantly with lack of self-control $\left(\mathrm{Rs}_{10}=0.77, p=0.01\right)$, reflecting the finding that ATD improved accuracy in anticipation of reward to a greater extent in more impulsive subjects (Figure 5). Post hoc correlational analysis revealed a nonsignificant negative correlation between lack of selfcontrol and reinforcement-induced improvement in accuracy following the balancing $\mathrm{TRP}+$ drink $\left(\mathrm{Rs}_{10}=-0.4\right.$, $p=0.3$ ), but a significant positive correlation between lack of self-control and reinforcement-induced improvement in accuracy following the depleting TRP - drink $\left(\mathrm{Rs}_{10}=0.77\right.$, $p=0.009)$. The difference between these two correlations was significant $\left(t_{7}=3.26, p<0.02\right)$, as revealed by a Williams test. Thus, the TRP-depleting drink induced a correlation between reinforcement-related accuracy and trait impulsivity that was not present following the control drink. Inspection of the graphs reveals that, relative to lowimpulsive subjects, high-impulsive subjects made more errors on high-reinforcement trials following the control drink. In contrast, following the TRP-depleting drink, highimpulsive subjects made relatively less errors on the same high-reinforcement trials compared with low-impulsive subjects.

The correlation between lack of self-control and the ATDinduced change in speeding on 90\%-reinforced trials relative to $10 \%$-reinforced trials tended toward significance $\left(\mathrm{Rs}_{10}=-0.56, p=0.095\right)$, as did the correlation between lack of self-control and change in accuracy $\left(\mathrm{Rs}_{10}=0.62\right.$, $p=0.058)$. There was no correlation between these BIS scores and the change in speeding on 50\%-reinforced trials compared with 10\%-reinforced trials (nonplanning: $\mathrm{Rs}_{10}=-0.03$; self-control: $\mathrm{Rs}_{10}=0$ ).

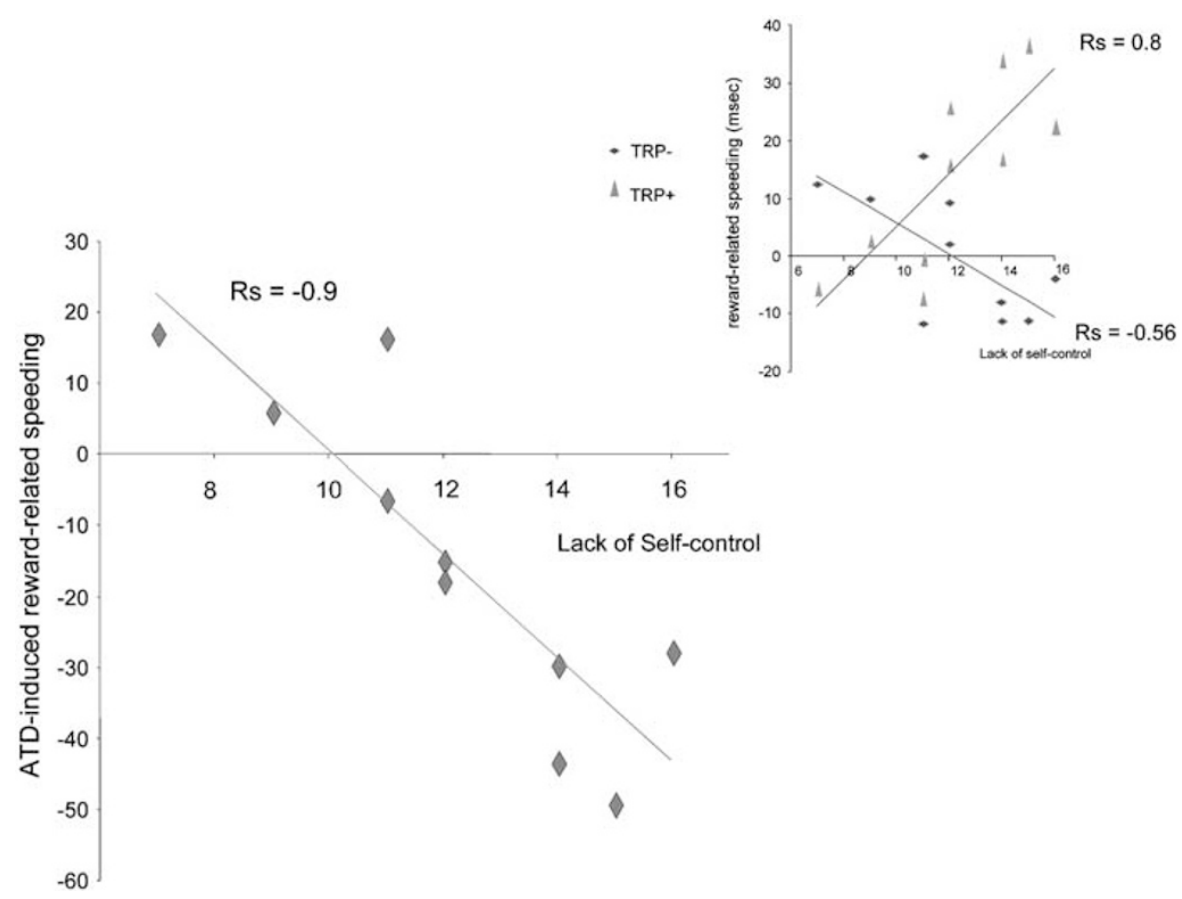

Figure 4 Inverse correlation between individual differences in trait impulsivity (lack of self-control) and changes in reinforcement-induced speeding, induced by ATD $\left(\mathrm{Rs}_{10}=-0.9, p<0.000 \mathrm{I}\right)$. ATD reduced speeding on $90 \%$-reinforced trials compared with $50 \%$-reinforced trials to a greater extent in high-impulsive subjects (with high scores on 'lack of self-control' factor III on the BIS-I I). Inset figure shows the data for each condition (TRP- and TRP + ) separately. 


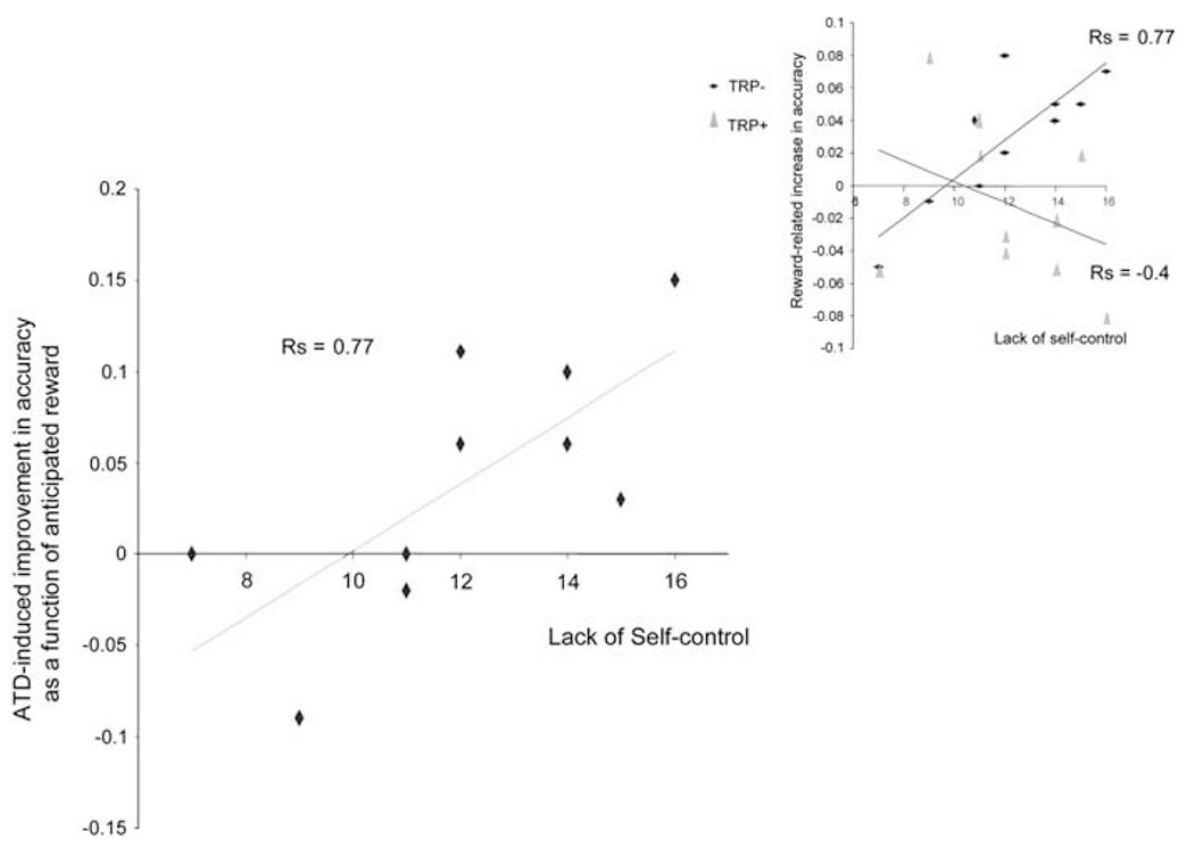

Figure 5 Positive correlation between individual differences in trait impulsivity (lack of self-control) and improved accuracy as a function of reinforcement certainty, induced by ATD $\left(\operatorname{Rs}_{10}=0.77, p=0.01\right)$. ATD improves accuracy on $90 \%$-reinforced trials compared with $50 \%$-reinforced trials to greater extent in high-impulsive subjects than low-impulsive subjects. Inset figure shows data for each condition separately.

Post hoc ANOVAs with the three first-order factors of the BIS-11 as covariates revealed a significant ATD $\times$ cue $(50 v s$ $90 \%)$ interaction $\left(\mathrm{F}_{1,8}=8.3, p=0.02\right)$ and a significant three-way interaction between ATD, cue $(50$ versus $90 \%) \times$ Nonplanning Impulsiveness $\left(\mathrm{F}_{1,8}=11.7, p=0.009\right)$, but not between ATD, cue, and any of the other two factors.

Supplementary analyses showed that the high correlation between trait impulsivity (lack of self-control) and the ATDinduced reduction in reinforcement-induced speeding was not contaminated by any ATD-induced difference in terms of mean overall RT. First, there was no effect of ATD on mean RT $\left(t_{9}=-0.1, p=0.9\right)$. Second, there was no correlation between the ATD-induced change in mean RT and the ATD-induced change in reinforcement-induced speeding $\left(\mathrm{Rs}_{10}=0.2, p=0.5\right)$. Third, the correlation between ATD-induced reinforcement-induced speeding and lack of self-control remained highly significant after correcting for ATD-induced differences in mean RT (partial correlation: $\mathrm{Rs}_{7}=-0.87, p=0.002$ ). Fourth, there was no effect of ATD on mean RT from the second practice block, which was used to calculate the cutoff score for determining reward magnitude (mean RT following TRP-: 641.9; TRP +: $\left.620.0 ; t_{9}=-0.5, p=0.6\right)$. An effect of ATD on mean RT from the practice block also did not correlate with reinforcement-induced speeding $\left(\mathrm{Rs}_{10}=0.3, p=0.4\right)$. The correlations between the effects of ATD and impulsivity were also not confounded by testing order as revealed by significant partial correlations between the ATD-induced effects and lack of self-control after correcting for testing order $\left(90-50 \%\right.$ speeding: $\mathrm{Rs}_{7}=-0.86, p=0.003 ; 90-50 \%$ accuracy: $\mathrm{Rs}_{7}=0.74, p=0.02$ )

In order to assess whether the change in performance as a function of reinforcement certainty, induced by ATD, was adaptive or maladaptive in the context of the CRRT, we correlated the total number of points obtained at the end of a block with performance change. There was a negative correlation between the total points score and the increase in accuracy as a function of reward probability $\left(\mathrm{Rs}_{10}=-0.83, p=0.003\right)$, but only in the TRP- condition, and not in the TRP + condition. Thus, following the TRPdepleting drink, but not the placebo drink, greater accuracy was associated with fewer points.

Debriefing. Analysis of debriefing data revealed that all subjects were explicitly aware of the associations between the different colors and reinforcement probabilities (main effect of cue: $\mathrm{F}_{2,18}=78.6, p<0.0001$ ), and ATD did not affect the ability to discriminate explicitly between colors at posttest (ATD $\times$ cue: $\mathrm{F}_{2,18}=0.4, p=0.6$ ). Analysis of confidence rating revealed that all subjects were more confident that their probability estimate was correct for the $90 \%$ and the $10 \%$ cues than for the $50 \%$ cues (effect of cue 90 vs $50 \%$ : $\mathrm{F}_{1,9}=9.4, p=0.014$ and cue 50 vs $\left.10 \%: \mathrm{F}_{1,9}=5.5, p=0.04\right)$. There was no significant effect of ATD (effect of ATD $\times$ cue 90 vs $50 \%: \mathrm{F}_{1,9}=0.7$ and cue 50 vs $10 \%: \mathrm{F}_{1,9}=0.2$ ).

\section{Stop-Signal Reaction-Time Task}

The within-subject analysis $(n=11)$ revealed no effects of ATD on the stop-signal task (SSRT: $t_{10}=-0.29, p=0.8$; GO RT: $t_{10}=0.7, p=0.5$; proportion of successful inhibitions: $t_{10}=-0.8, p=0.5$; discrimination error rate on GO trials: $t_{10}=-1.5, p=0.2$; see Table 1$)$. The between-subject analyses also revealed no effects of drink (data not shown).

Correlations. Following the placebo drink, response inhibition as measured with SSRT correlated with the secondorder BIS factor Motor Impulsiveness $\left(\mathrm{Rs}_{10}=0.72, p=0.01\right.$ ) (more impulsive subjects took longer to stop), but no 
Table I Mean Values from Within-Subject SSRT Analysis

\begin{tabular}{lcccc}
\hline & SSRT (ms) & $\begin{array}{c}\text { Median Go } \\
\text { RT (ms) }\end{array}$ & P inhibitions & $\begin{array}{c}\text { Mean Go } \\
\text { errors }\end{array}$ \\
\hline TRP- & 195.0 & 345.2 & 0.44 & 7.18 \\
TRP+ & 189.0 & 352.6 & 0.43 & 5.45 \\
\hline
\end{tabular}

correlations were observed between trait impulsivity and ATD-induced differences on the stop-signal task.

\section{Summary}

ATD slowed reaction times and increased accuracy on $90 \%$ reinforcement trials, relative to 50\%-reinforcement trials. These effects were greatest in subjects with high selfreported lack of self-control (Nonplanning Impulsiveness). In contrast, ATD did not affect response inhibition, which, on placebo, correlated with individual trait differences in Motor Impulsiveness.

\section{DISCUSSION}

Consistent with our prediction, 5-HT depletion disrupted motivated action by impairing the ability to adequately adapt responding to incentive-motivational cues signaling reinforcement certainty. Contrary to our expectations, 5-HT depletion did not affect response inhibition as measured with the SSRT.

Many of the effects of ATD at the (within-) group level were only marginal, perhaps reflecting the relatively modest sample size. However, the within-subject heterogeneity was resolved dramatically, and unexpectedly, when individual differences in trait impulsivity were taken into account. Thus, trait Non-planning Impulsivity correlated significantly with performance on the CRRT: Following the control drink, high-incentive cues induced the greatest speeding in subjects with high self-reported scores of lack of self-control. Most striking was the highly significant correlation between the effects of ATD on motivated action and trait impulsivity: central 5-HT depletion disrupted motivated action only in those individuals with great lack of self-control. While ATD abolished the correlation between reward-related speeding and trait impulsivity, it induced a significant correlation between reward-related improvements in accuracy and trait impulsivity.

The correlation is not contaminated by effects of ATD on the general speed of responding, as evidenced by significant partial correlations and an absence of a main effect of ATD on mean RT from both the practice and the experimental task. The data are also not confounded by practice or order effects, as suggested by a between-subject analysis of data acquired on a first session as well as significant partial correlations from the within-subject data set. Finally, effects cannot be explained by gross changes in mood and affect. Instead, the present findings suggest that ATD in young healthy volunteers disrupts motivated action depending on individual differences in trait impulsivity.

The strong dependence of the effects of ATD on individual differences in Non-planning Impulsivity ac- counts for the relatively small effects over the group as a whole, which comprised both low- and high-impulsive subjects. It is consistent with other work showing that ATD affects impulsive behavior only in certain individuals (Cherek and Lane, 1999; LeMarquand et al, 1999; Bjork et al, 2000; Marsh et al, 2002; Swann et al, 2002; but see Crean et al, 2002).

Following the placebo drink, high-impulsive subjects were more capable of adjusting their behavior adequately to cues predictive of reward than low-impulsive subjects. The increased speed of responding and decreased response accuracy could be interpreted as a risky, more impulsive strategy for high-reinforcement relative to low-reinforcement trials. Thus, it appears that on high-reinforcement trials, high-impulsive subjects recruited an excess of motivational processes leading to supra-accurate performance. Low-impulsive subjects appeared less sensitive to anticipated reinforcement certainty. We highlight two aspects of these findings in the 'placebo' subjects. First, in the context of this particular task, the impulsive strategy was a beneficial strategy: pay-off was greater for speed than for accuracy. While a fast correct response resulted in 100 points, a slow correct response resulted in 1 point and an incorrect response in 0 points. Second, the high-impulsive subjects only adopted such an 'impulsive' strategy on the $90 \%$-reinforced trials relative to the $50 \%$-reinforced, but not on the $50 \%$-reinforced relative to the $10 \%$-reinforced trials. Cues predictive of $50 \%$ reinforcement failed to push motivational processes above a certain 'supra-accurate threshold', the level of which appeared particularly dependent on trait impulsivity (and 5-HT levels).

It may be noted that the ATD-induced performance changes correlated more highly with trait impulsivity in terms of $90-50 \%$ differences than in terms of $90-10 \%$ differences. While there was very little reason for subjects to work at all for the $10 \%$ trials, recruitment of a moderate amount of motivational processes benefited performance on the $50 \%$ trials. Indeed, subjects made more errors on the $10 \%$ than the $50 \%$ trials. Perhaps, subjects were not fully focused on the task during the $10 \%$ trials and, therefore, the $50 \%$ trials may have provided a better motivational baseline for the comparison with the supra-accurate motivational state of the $90 \%$ trials.

The observed modulation of the present measure of motivated action by impulsive personality and ATD may reflect effects on a number of distinct underlying processes. For example, the performance profile in high-impulsive subjects may be due to reduced sensitivity to rewardpredicting signals and/or enhanced sensitivity to signals predicting punishment. Although speculative, this interpretation concurs with previous findings suggesting that impulsive people are less sensitive to punishment as well as more sensitive to reward (Wallace and Newman, 1990; Corr et al, 1997). By analogy, the ATD-induced performance changes on high-reinforcement trials in high-impulsive subjects may reflect a combination of reduced sensitivity to reward and an increased sensitivity to punishment. Indeed, Rogers et al (2003) recently reported that ATD impaired the ability to discriminate between large and small rewards. Diminished behavioral sensitivity to cues signaling reward and enhanced behavioral sensitivity to cues signaling punishment may parallel previous findings, indicating that 
depression, associated with diminished 5-HT function, is characterized by under-sensitivity to reward (Henriques et al, 1994) and over-sensitivity to negative feedback (Elliott et al, 1996, 1997; Murphy et al, 2002, 2003).

ATD did not affect SSRT, a measure of prepotent response inhibition. The negative result on SSRT in this small sample size (of 11 subjects) should be interpreted with caution and replication with a greater number of subjects should be awaited before firm conclusions can be drawn, particularly given a previous observation that ATD slowed SSRT in a group of 20 healthy men with a family history of alcoholism, while it improved SSRT in a group 20 healthy men without such a history (Crean et al, 2002). The discrepancy between the Crean et al study and our study may relate to the difference in sample size $(n=20$ in their study $v s n=11$ in our study) or the different dose: Crean et al administered $100 \mathrm{mg}$ drinks, associated with an average $84 \%$ reduction of TRP - levels relative to baseline, while we administered $75 \mathrm{mg}$ drinks, which reduced TRPlevels by $61 \%$ relative to baseline. Nevertheless, the differential sensitivity of the two measures observed here might be interesting in the context of their association with dissociable underlying corticostriatal circuitries (Taylor and Robbins, 1986; Jentsch and Taylor, 1999; Wyvell and Berridge, 2000; Eagle and Robbins, 2003a, b; Aron et al, 2003; Robbins, 2000).

The observed effect of ATD on behavioral sensitivity to cues predictive of reinforcement could be reinterpreted as an ATD-induced reduction in the adoption of a risky or impulsive strategy. This appears to contradict existing literature, suggesting that impulsivity implicates diminished 5-HT levels. However, the finding that 5-HT depletion in young healthy volunteers reduced active responding in anticipation of reward is consistent with more recent evidence that certain impulsive behaviors are associated with increased 5-HT levels (Puumala and Sirvio, 1998; Koskinen et al, 2000; Dalley et al, 2002; Passetti et al, 2003). Thus, Dalley et al (2002) revealed a positive correlation between premature responding on the five-choice reactiontime task in anticipation of reward and in vivo 5-HT efflux in the prefrontal cortex. Our data concur with the hypothesis that overactivation of 5 -HT receptors (in the PFC) may be an important mechanism that increases active responding, at least in anticipation of reward (Dalley et al, 2002).

In summary, together with the dissociable correlations between CRRT performance and Nonplanning Impulsiveness and between SSRT performance and Motor Impulsiveness, the present data highlight the multifactorial (neurobiological) nature of impulsivity and suggest that the 'diminished 5-HT hypothesis' of impulsivity is oversimplified. Our findings show that 5-HT depletion in healthy subjects disrupts motivated action as a function of anticipated reward probability and individual differences in the personality trait of impulsivity.

Future research is needed to assess whether parallel performance profiles are observed in people with depressive and/or impulsive symptomatology, and to what extent these may be normalized by 5 -HT-balancing drugs. The present findings suggest that certain vulnerable individuals are particularly likely to exhibit behavior, characteristic of both impulsive and depressive symptomatology, depending on acute TRP levels. Our findings may hold relevance to the observed comorbidity between depression, associated with loss of motivated action, and impulsive disorders (Deakin, 2003; Corruble et al, 2003).

\section{ACKNOWLEDGEMENTS}

We wish to thank Wim Riedel, Barbara Sahakian, Lisbeth Evers, Ed Bullmore, and Adam Aron for helpful assistance with the study. We also thank Mike Franklin and John Odontiadis, University of Oxford, for biochemical analyses. This work was supported by a Wellcome Trust Programme Grant to TWR (LC) and completed within the MRC Centre for Behavioral and Clinical Neuroscience. RC is a Royal Society Dorothy Hodgkin fellow and a Junior Research Fellow at St John's College Cambridge.

\section{REFERENCES}

Aron A, Fletcher PC, Bullmore ET, Sahakian BJ, Robbins TW (2003). Stop-signal inhibition disrupted by damage to right inferior frontal gyrus in humans. Nat Neurosci 6: 115-116.

Aronson SC, Black JE, McDougle CJ, Scanley BE, Jatlow P, Kosten TR et al (1995). Serotonergic mechanisms of cocaine effects in humans. Psychopharmacology 119: 179-185.

Bjork J, Dougherty D, Moeller F, Swann A (2000). Differential behavioral effects of plasma tryptophan depletion and loading in aggressive and nonaggressive men. Neuropsychopharmacology 22: 357-369.

Carpenter L, Anderson G, Pelton G, Gudin J, Kirwin P, Price L et al (1998). Tryptophan depletion during continuous CSF sampling in healthy human subjects. Neuropsychopharmacology 19: 26-35.

Cherek D, Lane S (1999). Effects of D, L-fenfluramine on aggressive and impulsive responding in adult males with a history of conduct disorder. Psychopharmacology 146: 473-481.

Cochran W, Cox G (1957). Experimental Designs. Wiley: New York.

Corr P, Pickering A, Gray J (1997). Personality and reinforcement in associative and instrumental learning. Person Indiv Diff 19: 47-71.

Corruble E, Benyamina A, Bayle F, Falissard B, Hardy P (2003). Understanding impulsivity in severe depression? A psychometrical contribution. Prog Neuropsychopharmacol Biol Psychiatry 27: 829-833.

Crean J, Richards J, de Wit H (2002). Effect of tryptophan depletion on impulsive behavior in men with or without a family history of alcoholism. Behav Brain Res 136: 349-357.

Dalley JW, Theobald DE, Eagle DM, Passetti F, Robbins TW (2002). Deficits in impulse control associated with tonically-elevated serotonergic function in rat prefrontal cortex. Neuropsychopharmacology 26: 716-728.

Deakin JF (2003). Depression and antisocial personality disorder: two contrasting disorders of 5HT function. J Neural Transm Suppl 64: 79-93.

Deakin JFW (1983). Roles of serotonergic systems in escape, avoidance and other behaviours. In: Cooper S (eds). Theories in Psychopharmacology. Academic Press: London and New York. pp 179-204.

Dougherty DM, Bjork JM, Marsh DM, Moeller FG (1999). Influence of trait hostility on tryptophan depletion-induced laboratory aggression. Psychiatry Res 88: 227-232.

Duncan J, Seitz R, Kolodny J, Bor D, Herzog H, Ahmed A et al (2000). A neural basis for general intelligence. Science 289: 457-460.

Eagle DM, Robbins TW (2003a). Inhibitory control in rats performing a stop-signal reaction-time task: effects of lesions 
of the medial striatum and D-amphetamine. Behav Neurosci 117: 1302-1317.

Eagle DM, Robbins TW (2003b). Lesions of the medial prefrontal cortex or nucleus accumbens core do not impair inhibitory control in rats performing a stop-signal reaction time task. Behav Brain Res 146: 131-144.

Elliott R, Sahakian B, Herrod J, Robbins T, Paykel E (1996). Neuropsychological impairments in unipolar depression: the influence of perceived failure on subsequent performance. Psychol Med 26: 975-989.

Elliott R, Sahakian B, Herrod J, Robbins T, Paykel E (1997). Abnormal response to negative feedback in unipolar depression: evidence for a diagnosis specific impairment. J Neurol Neurosurg Psychiatry 63: 74-82.

Evenden J (1999). Varieties of impulsivity. Psychopharmacology 146: 348-361.

Evers EA, Cools R, Clark L, van der Veen FM, Jolles J, Sahakian BJ et al (2005). Serotonergic modulation of prefrontal cortex during negative feedback in probabilistic reversal learning. Neuropsychopharmacology (Epub ahead of print).

Fletcher P, Korth K, Chambers J (1999). Selective destruction of brain serotonin neurons by 5, 7-dihydroxytryptamine increases responding for a conditioned reward. Psychopharmacology 147: 291-299.

Graeff FG, Brandao ML, Audi EA, Schutz MT (1986). Modulation of the brain aversive system by GABAergic and serotonergic mechanisms. Behav Brain Res 22: 173-180.

Harrison A, Everitt BL, Robbins TW (1997a). Central 5-HT depletion enhances impulsive responding without affecting the accuracy of attentional performance: interactions with dopaminergic mechanisms. Psychopharmacology 133: 329-342.

Harrison A, Everitt BL, Robbins TW (1997b). Double dissociable effects of median- and dorsal-raphe lesions on the performance of the five-choice serial reaction time test of attention in rats. Behav Brain Res 89: 135-149.

Harrison A, Markou A (2001). Serotonergic manipulations both potentiate and reduce brain stimulation reward in rats: involvement of serotonin-1A receptors. J Pharmacol Exp Ther 297: 316-325.

Henriques J, Glowacki J, Davidson R (1994). Reward fails to alter response bias in depression. J Abnorm Psychol 103: 460-466.

Howell DC (1997). Statistical Methods for Psychology. Wadsworth Publishing Company: Belmont.

Hughes J, Pleasants C, Pickens R (1985). Measurement of reinforcement in depression: a pilot study. J Behav Ther Exp Psychiatry 16: 231-236.

Jentsch JD, Taylor JR (1999). Impulsivity resulting from frontostriatal dysfunction in drug abuse: implications for the control of behavior by reward-related stimuli. Pharmacology 146: 373-390.

Kagan J (1966). Reflection-impulsivity: the generality and dynamics of conceptual tempo. J Abnorm Psychol 71: 17-24.

Koskinen T, Ruotsalainen S, Puumala T, Lappalainen R, Koivisto E, Mannisto P et al (2000). Activation of 5-HT2A receptors impairs response control of rats in a five-choice serial reaction time task. Neuropharmacology 39: 471-481.

LeMarquand D, Benkelfat C, Pihl R, Palmour R, Young S (1999). Behavioral disinhibition induced by tryptophan depletion in nonalcoholic young men with multigenerational family histories of paternal alcoholism. Am J Psychiatr 156: 1771-1779.

Liu Y, Wilkinson L, Robbins T (2004). Effects of acute and chronic buspirone on impulsive choice and efflux of 5-HT and dopamine in hippocampus, nucleus accumbens and prefrontal cortex. Psychopharmacology 173: 175-185.

Logan G, Cowan W, Davis K (1984). On the ability to inhibit simple and choice reaction time responses: a model and a method. J Exp Psychol Hum Percept Perform 10: 276-291.

Marsh D, Dougherty D, Moeller F, Swann A, Spiga R (2002). Laboratory-measured aggressive behavior of women: acute tryptophan depletion and augmentation. Neuropsychopharmacology 26: 660-671.

Mobini S, Chiang T-J, Ho M-Y, Bradshaw CM, Szabadi E (2000). Effects of central 5-hydroxytrytamine depletion on sensitivity to delayed and probabilistic reinforcement. Psychopharmacology 152: 390-397.

Murphy F, Michael A, Robbins TW, Sahakian BJ (2003). Neuropsychological impairment in patients with major depressive disorder: the effects of feedback on task performance. Psychol Med 33: 455-467.

Murphy FC, Smith K, Cowen P, Robbins TW, Sahakian BJ (2002). The effects of tryptophan depletion on cognitive and affective processing in healthy volunteers. Psychopharmacology 163: 42-53.

Nishizawa S, Benkelfat C, Young S, Leyton M, Mzengeza S, de Montigny C et al (1997). Differences between males and females in rates of serotonin synthesis in human brain. Proc Natl Acad Sci USA 94: 5308-5313.

Osman A, Kornblum S, Meyer DE (1990). Does motor programming necessitate response execution? J Exp Psychol Hum Percept Perform 16: 183-198.

Park S, Coull J, McShane R, Young A, Sahakian B, Robbins T et al (1994). Tryptophan depletion in normal volunteers produces selective impairments in learning and memory. Neuropharmacology 33: 575-588.

Passetti F, Dalley J, Robbins T (2003). Double dissociation of serotonergic and dopaminergic mechanisms on attentional performance using a rodent five-choice reaction time task. Psychopharmacology 165: 136-145.

Patton J, Stanford M, Barratt E (1995). Factor structure of the Barratt Impulsiveness Scale. J Clin Psychol 51: 768-774.

Puumala T, Sirvio J (1998). Changes in activities of dopamine and serotonin systems in the frontal cortex underlie poor choice accuracy and impulsivity of rats in an attention task. Neuroscience 83: 489-499.

Redgrave P, Horrell R (1976). Potentiation of central reward by localised perfusion of acetylcholine and 5-hydroxytryptamine. Nature 262: 305-307.

Reilly J, McTavish S, Young A (1997). Rapid depletion of plasma tryptophan: a review of studies and experimental methodology. J Psychopharmacol 11: 381-392.

Robbins TW (2000). Chemical neuromodulation of frontalexecutive functions in humans and other animals. Experimental Brain Research 133: 130-138.

Robbins TW, Cador M, Taylor JR, Everitt BJ (1989). Limbicstriatal interactions in reward-related processes. Neurosci Biobehav Rev 13: 155-162.

Robbins TW, Everitt BJ (2002). Limbic-striatal memory systems and drug addiction. Neurobiol Learn Mem 78: 625-636.

Robbins TW, Everitt BJ (2003). Motivation and Reward. In: Squire L, Bloom F, McConnell S, Roberts J, Spitzer N, Zigmond M (eds). Fundamental Neuroscience. Academic Press: London, UK. pp 1109-1126.

Robinson T, Berridge K (2003). Addiction. Annu Rev Psychol 54: 25-53.

Rogers RD, Blackshaw AJ, Middleton HC, Matthews K, Hawtin K, Crowley C et al (1999). Tryptophan depletion impairs stimulusreward learning while methylphenidate disrupts attentional control in healthy young adults: implications for the monoaminergic basis of impulsive behaviour. Psychopharmacology 146: 482-491.

Rogers RD, Turnbridge E, Bhagwagar Z, Drevets W, Sahakian B, Carter C (2003). Tryptophan depletion alters the decisionmaking of healthy volunteers through altered processing of reward cues. Neuropsychopharmacology 28: 153-162.

Sasaki-Adams D, Kelley A (2001). Serotonin-dopamine interaction in the control of conditioned reinforcement and motor behavior. Neuropsychopharmacology 25: 440-452. 
Soubrie P (1986). Reconciling the role of central serotonin neurons in human and animal behavior. Behav Brain Sci 9: 319-362.

Swann AC, Bjork JM, Moeller FG, Dougherty DM (2002). Two models of impulsivity: relationship to personality traits and psychopathology. Biol Psychiatry 51: 988-994.

Taylor JR, Robbins TW (1986). 6-Hydroxydopamine lesions of the nucleus accumbens, but not of the caudate nucleus, attentuate enhanced responding with reward-related stimuli produced by intra-accumbens d-amphetamine. Psychopharmacology 90: 390-397.

Wallace J, Newman J (1990). Differential effects of reward and punishment cues on response speed in anxious and impulsive individuals. Person Indiv Diff 11: 999.

Wilkinson LS, Humby T, Robbins TW, Everitt BJ (1995). Differential effects of forebrain 5-hydroxytryptamine depletions on Pavlovian aversive conditioning to discrete and contextual stimuli in the rat. Eur J Neurosci 7: 2042-2052.

Winstanley C, Dalley J, Theobald D, Robbins T (2003). Global 5HT depletion attenuates the ability of amphetamine to decrease impulsive choice on a delay-discounting task in rats. Psychopharmacology 170: 320-331.

Wogar M, Bradshaw C, Szabadi E (1993). Does the effect of central 5-hydroxytryptamine depletion on timing depend on motivational change? Psychopharmacology 112: 86-92.

Wyvell C, Berridge K (2000). Intra-accumbens amphetamine increases the conditioned incentive salience of sucrose reward: enhancement of reward 'wanting' without enhanced 'liking' or response reinforcement. J Neurosci 20: 8122-8130.

Young S, Smith S, Pihl R, Ervin F (1985). Tryptophan depletion causes a rapid lowering of mood in normal males. Psychopharmacology 87: 173-177. 